\title{
Philosophiques
}

\section{Les avatars de la différence}

\section{Michèle Morosoli}

Volume 11, numéro 2, octobre 1984

Égalité, justice et différence

URI : https://id.erudit.org/iderudit/203265ar

DOI : https://doi.org/10.7202/203265ar

Aller au sommaire du numéro

\section{Éditeur(s)}

Société de philosophie du Québec

\section{ISSN}

0316-2923 (imprimé)

1492-1391 (numérique)

Découvrir la revue

\section{Citer cet article}

Morosoli, M. (1984). Les avatars de la différence. Philosophiques, 11(2), 389-413. https://doi.org/10.7202/203265ar

\section{Résumé de l'article}

L'égalité générale, c'est-à-dire l'égalité de tous envers tous, même si elle est préjugée favorablement, est difficilement réalisable à cause de notre pensée qui organise les différences en dichotomies et en oppositions hiérarchisées. Quand on examine des discours écrits par des femmes cherchant à définir la différence de la femme, on aperçoit de manière patente cette difficulté à nommer la différence. En comparant un texte de E. Harding, psychanalyste jungienne et un texte de M. Montrelay, psychanalyste lacanienne, on constate que malgré des provenances théoriques et historiques éloignées, le féminin n'est dit qu'en référence au masculin. Avec notre mode de pensée (qui convertit les différences en des oppositions hiérarchisées), le désir d'égalité générale s'avère vélléitaire. Seul un discours éclaté qui donne lieu aux différences permettrait une mentalité égalitaire.
Tous droits réservés (C) Société de philosophie du Québec, 1984
Ce document est protégé par la loi sur le droit d'auteur. L'utilisation des services d'Érudit (y compris la reproduction) est assujettie à sa politique d'utilisation que vous pouvez consulter en ligne.

https://apropos.erudit.org/fr/usagers/politique-dutilisation/ 


\title{
LES AVATARS DE LA DIFFÉRENCE
}

\author{
par Michèle Morosoli
}

RÉSUMÉ. L'égalité générale, c'est-à-dire l'égalité de tous envers tous, même si elle est préjugée favorablement, est difficilement réalisable à cause de notre pensée qui organise les différences en dichotomies et en oppositions hiérarchisées. Quand on examine des discours écrits par des femmes cherchant à définir la différence de la femme, on aperçoit de manière patente cette difficulté à nommer la différence. En comparant un texte de E. Harding, psychanalyste jungienne et un texte de M. Montrelay, psychanalyste lacanienne, on constate que malgré des provenances théoriques et historiques éloignées, le féminin n'est dit qu'en référence au masculin. Avec notre mode de pensée (qui convertit les différences en des oppositions hiérarchisées), le désir d'égalité générale s'avère vélléitaire. Seul un discours éclaté qui donne lieu aux différences permettrait une mentalité égalitaire.

ABSTRACT. Equality, when it is understood as equality for all, even though it is judged favorably in general, is hardly realisable because of the structure of our thinking that organizes the differences in dichotomies and in hierarchical oppositions. When we examine the discourses written by women that tend to define a difference of the woman, we realize the difficulty to name this difference. When we compare a text written by E. Harding, a jungian psychanalyst and another written by M. Montrelay, a lacanian psychanalyst, we observe that despite their distant theorical and historical sources, the feminine is said in reference to the masculine. By our way of thinking (that converts all differences in hierarchical oppositions), the desire for a generalized equality becomes erratic. Only a fragmented discourse, that gives place to the differences could permit an equality in the mentalities.

L'égalité politique, l'égalité légale ou l'égalité économique appartiennent aux égalités qui ont la chance de pouvoir être quantifiables, qui bénéficient de l'avantage de pouvoir être pensées en systèmes applicables. Elles semblent donc plus facilement 
réalisables que l'égalité générale, cette vague et vaste valeur pour laquelle toute notre époque se prononce d'emblée : l'égalité de tous envers tous. Elle peut s'entendre comme la prescription suivante : tout homme, quel qu'il soit, a droit à une reconnaissance morale, humaine égale, quelle que soit sa situation sociale, économique ou psychologique.

Cependant si on en examine les possibilités réelles d'enracinement, elles me semblent minces. En effet, ce préjugé favorable à l'égalité cohabite avec les multiples préjugés défavorables à toutes les différences. Ces préjugés sont solidement établis par une manière de penser à l'aide d'oppositions d'ores et déjà hiérarchisées et par une manière d'imaginer à travers des représentations conscientes et inconscientes marquées par des millénaires d'inégalités.

Ce mode de pensée permet des économies intéressantes : l'économie de la connaissance du multiple et de toutes les menaces qu'il recèle, l'économie des discours au profit du seul discours de l'Un, de la seule référence établie. Elles servent à renforcer le pouvoir.

Je crois que l'exemple de la femme peut mettre à jour de façon particulièrement probante les économies de cette pensée difficilement susceptible de nommer en un sens égalitaire.

Dans un premier temps, je cherche à le démontrer en analysant les métaphores parlant le féminin de deux psychanalystes d'écoles bien différentes : Esther Harding, psychanalyste jungienne, ayant publié en 1953 un ouvrage intitulé Les mystères de la femme et Michèle Montrelay, psychanalyste lacanienne, ayant publié en 1977 une étude sur la sexualité féminine, L'Ombre et le Nom. Les métaphores employées par chacune, nous le verrons, sont nettement marquées par tout un jeu d'oppositions hiérarchisées. Par la suite, j'examine la proposition de Luce Irigaray et de Jacques Derrida à l'effet de subvertir la pensée, de la déconstruire, de l'amener dans des lieux échappant à la nomination inégalitaire des choses. Finalement, j'examine si la proposition d'élaborer un discours multiple est suffisante pour amener un discours égalitaire. 
UN COUPLE : LUNE/SOLEIL

Il y a trente ans déjà, Esther Harding ${ }^{1}$ nous proposait de lire lés mystères de la femme à travers les différents mythes, légendes et rêves qui parlent de la lune. La lune est un vieux symbole que l'on retrouve de manière presque universelle dans toutes les mythologies. Chaque fois il représente la féminité sous un aspect ou un autre. En se penchant sur ces mythes et légendes, les femmes pourront ne pas perdre le sens du principe féminin, sens d'autant plus important à notre époque que la connaissance s'éloigne de plus en plus de la saisie subjective des objets, pour se centrer sur une forme qui se veut uniquement objective. Le délaissement de cette forme de connaissance est dommageable et pour l'homme et pour la connaissance, mais encore plus pour la femme, son lieu étant le subjectif. Aussi, les femmes ont délaissé les rôles féminins traditionnels pour explorer certains rôles masculins. De là, l'urgence à ce que la femme apprenne à vivre selon ses principes masculins et féminins.

Avant de s'intéresser à sa présentation des différents mythes lunaires, il faut éclaircir quelque peu les positions théoriques de E. Harding. C'est une psychanalyste jungienne et sa vision de la femme et du mythe est déterminée par l'appareil théorique de Jung. Considérons quelques-unes de ses positions, en particulier celles qui touchent à la Nature et aux archétypes.

Jung postule fortement l'existence d'une Nature. Elle serait vraie, juste, guide; une analyse mènerait à saisir dans l'inconscient son essence, permettrait d'atteindre sa vérité. Bipolaire, cette Nature est composée de contraires opposés : la naturel'esprit ; l'humain-l'inhumain ; le conscient-l'inconscient ; l'extraversion-l'introversion ; la pensée-le sentiment ; le dedansle dehors ; le clair-l'obscur ; le yin-le yang. Cet contraires peuvent s'harmoniser par une conjonction, mais ils restent toujours distincts.

Quant aux archétypes, ils sont " la nature à l'état non faussé ${ }^{2}$. À la fois images et émotions, ils sont les résidus d'ex-

1. Harding, Esther, Les mystères de la femme, Paris, (Petite Bibliothèque Payor), 1976.

2. Voir Baudoin, Charles, L'ceuvre de Jung, Paris, (Petite Bibliothèque Payot), 1975, p. 367. 
périences plusieurs fois millénaires de lutte pour l'adaptation et l'existence. Ils n'ont pas de contenu déterminé, mais ils sont influencés par les cultures, les époques. Ils ne sont déterminés que dans leur forme et à un degré limité comme le système axial d'un cristal : il préforme la structure cristalline dans l'eau-mère mais n'a aucune existence matérielle. Bipolaires, ils possèdent un côté sombre et clair, un aspect redoutable et salutaire et ils ramènent aux lois immuables de l'univers. Ils surgissent dans les rêves, les symboles, les mythes, les légendes. On peut qualifier cette notion de platonicienne : la psyché n'est pas vide tabula rasa - mais susceptible de produire des formes qu'elle contient.

Il existe plusieurs archétypes dont l'animus (principe mâle) et l'anima (principe femelle), couple représentant la plus haute forme d'opposition des contraires. Tout homme possède en lui une composante féminine, une âme, un souffle qui est le conglomérat de toutes les expériences de la lignée ancestrale au sujet de l'être féminin. Cette anima s'incarne dans différents personnages ; ils sont multitude mais, entre autres, on peut recenser la femme fatale, la jeune fille, la femme inspiratrice, la nymphe, l'ondine, la sirène, la femme-cygne, la lune. Cette composante baigne dans l'inconscient masculin. Elle peut s'harmoniser à la personnalité mais ne peut jamais être tout à fait conquise. De même la femme possède une composante masculine, baignant dans l'inconscient, l'animus qui peut se manifester à travers les images suivantes : l'étranger, l'inconnu, le magicien, le gardien, le chef, le sage.

Et l'homme et la femme doivent tendre à s'adjoindre leur partie inconsciente mais sans toutefois pouvoir les vivre dans une égalité. La conjonction des contraires se fait dans une tentative de complémentarité ; celle-ci respecte les différences et les hiérarchise. On ne se retrouve pas en face de l'androgyne qui est tour à tour autant mâle que femelle.

Harding se penche sur des mythes qui abondent en archétypes pour saisir la psyché, et plus particulièrement sur les mythes lunaires qui permettent de revenir au principe féminin. Le principe est une loi essentielle, intérieure ; il s'agit de le retrouver. Il faut donner ici au mot loi la même acception qu'en sciences positives. 
Pourquoi a-t-on signifié, pourquoi signifier encore le masculin par le soleil, le féminin par la lune ? Esther Harding ne pose pas la question et accepte ce symbolisme comme évident. "Le fait que le soleil semble à l'humanité le symbole du mâle tandis que la lune symbolise la femelle se comprend aisément $»^{3}$. Et suivent les caractéristiques de l'un et de l'autre. Évidemment, ces analogies sont discutables.

À l'aide des tableaux suivants ${ }^{4}$, je cherche à mettre en relief les oppositions employées par Harding et à souligner combien elles connotent les différences du masculin et du féminin d'une inégalité constante. L'exposé de Harding semble dire : le féminin a telle qualité ; si on la compare à la qualité masculine, elle se révèle être un défaut ; si on se place du côté du désir masculin ce défaut peut être rétabli comme une qualité ; cependant elle reste dangereuse.

Par exemple - on peut ressentir ce typè de présentation dans tous les éléments du tableau ; je choisis le premier - : la lune/féminin est changeante (qualité) ; le soleil/masculin, lui, est une source constante et sûr de la lumière et de la chaleur, ce qui est beaucoup plus rassurant et bénéfique. Mais le soleil/ masculin, dans sa constante régularité, éprouve cette imprévisibilité de la lune/féminin comme un mystère et alors par le biais de sa curiosité elle devient une qualité mais toujours un peu suspecte.

SOLEIL

1. Source constante et sûre de la lumière et de la chaleur.
LUNE

1. Changeante.

Le soleil a un cycle journalier, saisonnier qui affecte sa constance.

Intempéries, phénomènes naturels (nuages, éclipses) affectent la perception de sa régularité.

3. Harding, Esther, op. cit., p. 74.

4. Les citations du tableau se retrouvent dans les pages 74 à 92 dans le livre Les mystères de la femme. 
La lune a un cycle de 28 jours, cycle quaternaire : pleine lune et lune noire, croissance et décroissance.

Somme toute, le cycle de la lune est aussi régulier, aussi aléatoire que celui du soleil ...

2. Ou bien présent ou bien absent (univoque).

Brille, disparaît, réapparaît (prévisible).
2. Parfois présente, parfois absente comme par caprice (plurivoque).

Parfois brille, parfois pas (imprévisible).

"Parfois le midi présence spectrale qui semble une protestation contre la lumière éclatante et brutale du soleil ».

Il faut admettre à la lune un cycle plus complexe qu'au soleil. Mais à quoi tient toute cette interprétation? À des projections psychologiques faites sur ces astres? Certes, mais ces projections expriment-elles un ordre naturel, une organisation biopsychologique, la perception que l'homme a de lui-même et de la femme - si l'on affirme que mythes et légendes sont produits par les hommes - l'ordre socioéconomique ou encore . . . ?

3. Jésus-Christ, « dont le splendide rayonnement illumine le juste qui vit dans la lumière de la Grâce ».
3. Marie, « dont le doux éclat illumine les pécheurs au milieu de la triste nuit du péché ».

Peut-être est-ce le lieu, où l'on sent le plus, à notre époque laïque, le plaisir-désir de définir des termes par l'opposition, par l'emploi de l'axe de symétrie . . . 
4. Le soleil brille par sa lumière.
4. La lune brille du reflet de sa lumière.

Quand cette connaissance-là a-t-elle été acquise ? Cette analogie semble permettre de pointer le travail de construction de l'interprétation.

5. Force lumineuse chaude et créatrice.

5. Le plus grand principe de transformation par les choses les plus basses.

Ce qui est sombre, froid, humide, ce qui échappe à la lumière du jour contient également le secret de la vie.

De l'animal à l'homme? De l'instinct au spirituel ? De la nature à la culture ? Pourquoi imaginer déjà à l'origine des choses une hiérarchisation?

Harding ajoute une autre opposition, celle du Logos/Eros.

SOLEIL/LOGOS

1. Le jour.

2. La conscience.

3. Le travail et son accomplissement.

4. La compréhension directe et la discrimination.

5. La constance, la loi.
LUNE/EROS

1. La nuit.

2. L'inconscient.

3. Une puissance fatale à l'œuvre.

4. L'incompréhension, l'obscur.

5. L'inconstance, le chaos.

Le lieu du pouvoir - l'échappé du pouvoir circonscrit par sa compréhension directe et discriminatoire?

Établissement de l'opposition ; ce qui permet l'équilibre et alors facilite un certain exercice du pouvoir? 
Harding désigne une troisième opposition qui permet de connaître le masculin/féminin :

SOLEIL/LOGOS/YANG

1. Ce qui apparaît, se dit.

2. Force lumineuse, chaude, créatrice.
LUNE/EROS/YIN

1. « L'essence féminine quand on en parle n'est plus la véritable essence féminine ».

2. Élément sombre, obscur, réceptif et créateur car il produit et manifeste tous les élans créateurs de l'énergie Yang.

La première naissance, celle de l'obscurité donnant le corps, la nature, l'instinct, estelle ignoble ? Et la seconde naissance, celle de la lumière, donnant l'esprit, la culture, le spirituel; est-elle noble ? Pourquoi cette reconnaissance en ces termes? Jusqu'à quel point se fait-elle au détriment de la première naissance, de la mère, de l'origine (cf. Luce Irigaray)?

E. Harding ne songe jamais à remettre en question ces expressions du principe féminin. Elles sont vraies parce qu'archétypales (donc universelles et numineuses). Il faut cependant lire ces archétypes avec l'esprit du siècle, les suivre en les revivifiant. E. Harding est féministe en ce sens qu'elle demande pour la femme une autonomie psychologique et économique ; qu'elle valorise le contact de la femme avec le féminin - contact plus propice, par exemple, au temps des menstruations qu'il ne faut pas mépriser mais dont il faut entendre les " enseignements ». Mais son féminisme est modéré, la femme devant garder son rôle, la place que la Nature lui a assignée.

Cette relecture des mythes est assez moralisante ; y est toujours sous-jacente l'intention d'y trouver un message, une révélation, un guide . . . Quelle légitimité peut acquérir un tel traitement du mythe ? Cette relecture ne manque pas d'être 
agaçante par le cachet vieillot qui s'en dégage. Les mœurs ont tendance à vieillir et l'écart de trente ans écoulé entre le temps de sa publication et aujourd'hui se fait ressentir, me semble-til.

En outre, cette manière de traiter le mythe, de poser l'archétype comme source de vérité repose l'antique question de l'existence de la morale naturelle. Y aurait-il quant à l'agir des principes aussi inévitables que des lois qui auraient force de contrainte pour la plupart ? ou pour les plus inconscients? ou pour les analysés ? Et dans quelle mesure cette morale naturelle peut-elle échapper aux transformations culturelles, idéologiques? Dans quels lieux ne le peut-elle pas ?

De même on se retrouve devant la question épineuse de la nature contre la culture. D'où naissent les déterminations du féminin ? Peut-on dire qu'il est nature, corps, ombre et que toute intériorisation permettra de le récupérer dans la perte qu'il a subie lors de la période patriarcale ? Est-il déjà inscrit d'une façon si forte, si déterminante qu'il fait figure de nature ? Peutil encore s'inventer?

On peut poser une question d'ordre politique plutôt qu'épistémologique : - dans ce genre de débat, l'intérêt est si manifeste qu'il doit parfois présider à la construction, à la destruction de discours (?) - quelle est la valeur pour la femme de la conception jungienne du monde ? Le monde est composé de couples de contraires ; ils s'articulent sur le modèle d'une positivité et d'une négativité. La femme appartient au pôle infériorisé. Dans un tel contexte aucune revendication égalitaire ne peut prendre sens puisqu'elle vise l'utopie . . . Alors comment s'attacher à ces dires, surtout si l'on est femme ?

Pourtant, E. Harding, à travers une très forte documentation, décrit le principe féminin dans l'intention de permettre à la femme de se conscientiser, travail qu'elle juge primordial à notre époque. À cause de sa nature la femme est plus proche de l'inconscient, de l'instinct, de l'animal. Dans cette sphère les forces sont bipolaires et sauvages, créatrices et démoniaques. Mais dans un moment de déséquilibre, de transformations, les forces destructrices tendent à l'emporter et atteignent des sommets inégalés. La femme doit donc s'occuper activement d'ame- 
ner à la conscience ses forces obscures afin de mieux prendre sa place dans la société (elle a été dévalorisée, mise au second rang par le système patriarcal), mais elle doit prendre sa juste place et non une place usurpée dans la colère de la réaction.

Toute cette conception, toute cette prudence pour maîtriser des forces qui se révéleraient destructrices, ne sont-elles pas engendrées par la peur? Peur de l'homme, de la femme pour la femme. Gammes de peurs introjectées et la peur, notamment, du refoulé.

Le féminin serait-il nommé du lieu de la peur?

\section{UN AUTRE : OMBRE/NOM}

Dans un livre plus récent (1977) Michèle Montrelay, psychanalyste lacanienne, aux antipodes d'Esther Harding, parle de la sexualité féminine et du féminin en effectuant une synthèse des discours analytiques courants - ce qui amènerait la fin d'oppositions qui n'ont pas lieu d'être - et en proposant des métaphores nommant le masculin et le féminin.

Examinons si l'on échappe aux oppositions que je suspecte d'être toujours le lieu de l'inscription inégalitaire. Sera-t-il parlé du féminin de manière à ce qu'il puisse être pensé égal ?

Montrelay ${ }^{5}$ constate que pour parler de la question de la féminité, on se réfere à la vieille polémique Freud-Jones et on se range soit dans un camp, soit dans l'autre. Rappelons brièvement ces positions. Pour Freud, il existe une libido, et une seule, d'essence mâle ; et la sexualité féminine s'élabore à partir de repères phalliques (clitoris-pénis ; enfant-pénis). Jones, au contraire, affirme une libido spécifiquement féminine ainsi qu'une sexualité féminine subissant très tôt l'impact de l'anatomie et s'organisant autour de repères oraux-anaux-vaginaux.

M. Montrelay suggère un nouveau point de vue qui maintient la vérité de deux écoles mais permet d'en dépasser la querelle : phallocentrisme et concentricité sont également constitutifs de la sexualité féminine; cependant ils coexistent dans

5. Montrelay, Michèle, L'Ombre et le Nom, Paris, (Minuit), 1977. 
l'inconscient féminin comme incompatibles. Cette incompatibilité est spécifique à l'inconscient féminin.

Ce point de vue prend source dans un problème concret auquel l'analyse se bute : l'immobilisme féminin. Cet immobilisme peut être imputé à " l'envie du pénis ». Certaines femmes se maintiennent en état d'impuissance et affirment qu'il provient de la non-possession du pénis. Cette attitude institue le pénis comme valeur inestimable. Il faut cependant entendre cette " envie du pénis » comme désir du phallus, c'est-à-dire comme un désir construit autour du représentant privilégié du désir (explication liée à la thèse de Freud).

Cet immobilisme peut aussi s'expliquer à partir des expériences archaïques de la féminité (explication liée à la thèse de Jones). On a dit de la sexualité féminine - et on l'a beaucoup redit - qu'elle était un continent noir. On peut croire : parce que pas encore explorée, ou parce que inexplorable. Montrelay interprète cette parole dans le second sens proposé. Si on écoute la vie d'un discours d'analysante, on la voit se donner comme contenu manifeste, sous un mode immédiat ; rien de latent. Fait défaut au moins partiellement le refoulement dans le discours et aussi dans la sexualité féminine. C'est donc à cause de son rapport spécifique à la représentation que la sexualité féminine est « ininterprétable. »

En principe, la représentation prend place quand il y a perte de jouissance. Ou encore le refoulement, quand il s'opère, dessaisit l'objet de sa jouissance, la place dans la représentation, c'est-à-dire dans le mot investi au détriment de la réalité. L'enjeu est toujours relancé par la représentation même qui le récupère. La représentation est donc castratrice.

Chez la femme, cependant, a lieu la faillite de la représentation. La représentation se produit sans doute, mais elle ne détourne pas le sujet de la jouissance, le réel s'imposant dans son immédiateté. Aussi, elle se produit moins, la féminité étant plus sujette à la censure qui se présente comme un blanc, une absence de représentation, un manque. La censure est donc irreprésentable, ininterprétable.

Cet échec de la représentation est dû à plusieurs causes. 
L'absence d'interdits : la non-visibilité du sexe féminin protège la petite fille des interdits de la masturbation. L'enjeu de la castration se trouve déplacé sur l'autre sexe.

L'oralité : la sexualité féminine tourne autour d'un « organetrou-insatiable qui infléchit tout mouvement psychique selon des schèmes circulaires et clos. Ce mouvement compromet le rapport de la femme à la castration et à la loi. Son expérience érotique réactive un rapport au monde captateur, enveloppant ; son rapport au discours est de le piéger, de l'attirer vers elle alors que l'homme le respecte comme instance déterminante.

L'inceste : le rapport à son corps et non seulement narcissisant mais aussi érotique, voire incestueux. Chaque rapport à son corps la met en présence du premier objet le corps (phallus) maternel. La violence du réel (présence récupérée du corps maternel lors de tout contact à son corps propre) trouble l'économie de la représentation. Elle l'empêche.

Ainsi M. Montrelay pose la question : si la menace d'une possible castration est loin d'être patente, si les interdits ne fonctionnent pas assez fortement pour obliger à abandonner les plaisirs infantiles, si l'érotisme adulte réactive les jouissances premières, comment la femme peut-elle s'assurer de la castration symbolique?

Puisque l'enjeu manque, l'économie du désir et de la représentation est toujours à refaire. Et elle le met en représentation, ce manque, à travers la mascarade, le travestissement de son corps qui lui justement est image de cette castration. Voilà pourquoi on dit depuis toujours qu'elle incarne le mal. À cause de la confusion faite entre son corps qui montre la castration et son désir qui est insuffisamment marqué de la castration, le symbolique est ébranlé. De là, l'angoisse et les accusations de l'homme envers la femme.

«En d'autres termes, la féminité « selon Jones », vécue sur un mode réel et immédiat, fait tache aveugle dans les processus symboliques analysés par Freud. Deux territoires hétérogènes, incompatibles, coexistent à l'intérieur de l'inconscient 
féminin : celui de la représentation, celui qui reste " continent noir $» »^{6}$.

Il s'ensuit que l'on retrouve dans l'inconscient féminin deux sortes d'économie. Elles coexistent " avec prédominance (provisoire ou définitive) de l'une d'elles ${ }^{7}$.

Tantôt (économie selon Jones) l'enjeu est le phallocentrisme, le représentant est l'orifice vagino-oral. Le rapport à la jouissance est un rapport d'angoisse car la jouissance renforce le rapport au corps propre.

Tantôt (économie selon Freud) l'enjeu est la féminité précoce, le représentant le phallocentrisme. Le rapport à la jouissance serait un rapport de sublimation.

M. Montrelay donne à la sublimation une nouvelle acception : elle a lieu quand deux signifiants sont articulés, quand le refoulement a enfin lieu pour que la symbolisation puisse prendre place.

Le saut décisif de l'inconscient féminin se trouve déplacé : il ne réside pas dans le changement d'objet (de la mère au père) mais dans le changement du représentant (de la concentricité au phallus).

Et M. Montrelay soulève la question : la psychanalyse se serait-elle articulée pour refouler la féminité c'est-à-dire en faire de la représentation symbolique?

Montrelay pose la coexistence incompatible de deux représentants, de deux économies comme spécifique à l'inconscient féminin. Elle cherche à résoudre des querelles d'écoles qui affirment des oppositions exclusives. Une théorie n'abolit plus l'autre mais la chevauche. Est-ce affirmer le pluriel ? sortir des oppositions?

Aussi elle propose un dépassement du cercle de la féminité. L'organe véritable de jouissance chez la femme est beaucoup plus que telle ou telle partie du corps, le signifiant. La « vraie » femme, celle qui aurait cessé de toujours vouloir être son sexe

6. Montrelay, Michèle, op. cit., p. 70.

7. Idem, p. 74 . 
confierait sa féminité à la représentation, à la métaphore, à l'articulation que peut en faire le sexe masculin. Ce serait à lui de dire, de nommer la féminité.

Ou encore parlons de cela à travers deux métaphores qu'elle nous propose : l'Ombre et le Nom.

L'Ombre peut être dite jouissance de la Mère, le Nom jouissance du Père. Qu'est-ce qui les distingue ?

L'Ombre : en elle a lieu la « première " perte de la vie mais en elle, la vie est préservée. Elle donne consistance à la perte et aussi permet que l'enfant naisse au désir. D'elle on ne peut pas dire grand-chose car elle est le lieu du silence. Certes, elle possède ses signifiants mais ils sont refoulés d'un refoulement primaire, à jamais inconscients, envahis par la nuit des temps. On peut rentrer en contact avec eux mais pas les parler puisque cette jouissance abolit toute pensée : elle ouvre sur un oubli infini, sur une jouissance infinie, incommensurable.

Lieu de la rencontre à la Mère, la femme entretient avec l'Ombre des complicités qui lui permettent le contact avec la féminité, la maternité. Contact différent selon que la femme donne naissance à un garçon : "il fait émerger et réinsère dans l'ordre visible, sûr de la réalité et des objets ${ }^{8}$; ou à une fille : "ça demeure dans l'obscur " ${ }^{9}$, ça reste dans les limbes.

En elle, la femme se perd, se quitte, saisie par la jouissance de l'Autre, "pas-toute ", dans l'Autre là où on ne peut être qu'absent.

Le Nom : ce qui sort de l'Ombre, l'ayant entrouverte, désignant la chose, la nommant, la poinçonnant à ses armes. Jouissance entière, finie, organisée autour de son attirail de mots et de noms, non trouée ; elle fait sens car elle articule, permet des signifiants repérables. Elle assigne un lieu à l'Autre, "inscrit sur lui, en lettres réelles, sa trace ${ }^{10}$.

Lorsque le Nom surgit, il refoule, organise, imprime la sexualité archaïque de la Loi.

8. Idem, p. 140.

9. Ibidem.

10. Idem, p. 144. 
Sur quelles voies ces métaphores nous induisent-elles? Quelles sont les connotations qu'elles évoquent ? Quelque part je me sens ramenée aux mythes, légendes et rêves commentés par E. Harding. Évidemment pas de traces chez M. Montrelay d'une morale métaphysique qui nous presse de trouver la vérité, ni d'une Nature qui nous demande d'harmoniser en nous ses contraires, ni d'essences qu'il s'agit de dévoiler. Le panorama de fond est différent : l'inconscient est langage, articulations de signifiants. Il faut en écoutant les discours des analysantes décrypter le refoulé/non refoulé, le censuré de la femme, porter au dire, à la représentation. Mais il n'en reste pas moins que ces métaphores renvoient soit explicitement, soit dans leurs connotations à celles d'E. Harding.

Essayons de le montrer à l'aide du schéma suivant ${ }^{11}$ :

LE NOM

LIEU DE LA JOUISSANCE

DU PÈRE

1. lieu de la désignation

- ce qui joue des mots et des noms

- nomme

- assure « l'insertion dans la réalité »
L'OMBRE

LIEU DE LA JOUISSANCE

DE LA MÈRE

1. lieu du silence

- indicible

- puisque ses signifiants sont « refoulés d'un refoulement primaire »

- puisqu'en se produisant « elle abolit toute pensée » parole (?).

\section{(Culture/Nature ?) \\ (Logos/Éros ?) \\ (Pensée/Sentiment ?)}

2. clair

- le visible
2. « obscure»

- lieu de «la Nuit », du « refoulé d'un refoulement primaire».

11. Dans ce tableau apparaît un vocabulaire qui court tout au long du livre. On trouvera cependant une description plus serrée de l'Ombre de la page 138 à la page 140, et du Nom de la page 143 à la page 146. 
- qui laisse des traces dans le réel

- le fini
- les « limbes » qu'on ne peut quitter

- lieu de l'infini, l'incommensurable

(Soleil/Lune ?)

(Lumière/Ombre ?)

(Logos/Éros ?)

3. ce qui ordonne

- donne lieu, nom aux objets
3. « un lieu silencieux et mobile, jonché de débris, balayé de courants où les éléments se mélangent, se propagent pour se diviser brusquement traversés par quelles forces?»

(Cosmos/Chaos?)

(Ordonnancement fort du Logos/Force vive de la Nature ?)

4. ce qui «expulse la chose"

- « nomme»

4. prend « corps ", « s'actualise "

- " s'incarne charnellement »

(Esprit/Corps ?)

5. ce qui « expulse la chose» 5. « cette substance féminine - «blesse " l'infini

- l'« agresse " de jouissance qui fait étoffe à l'Autre, l'enveloppe et le développe à l'infini "

(Activité/Passivité ?)

(Agressivité/Réceptivité ?)

6. «Que celui qui jouit parle et nomme, c'est cela qui fait trauma et marque à la fois. La jouissance non trouée, tout d'une pièce, $\mathrm{du}$ père, en assignant un lieu à l'Autre, attaque son infini, le blesse. Mais en le blessant, inscrit sur lui en lettres réelles sa trace ».
6. fait naître au corps, support de la naissance du désir - donner essor 
(Le premier engendrement : celui du corps, de la matière qui sera informée de tous les potentiels à être réalisés par le second engendrement celui du Père, du Nom, du Logos, engendrement noble car il donne forme ?)

On se retrouve face à des couples d'opposés métaphorisés et connotés de manière assez traditionnelle. Cela apparaît nettement si on essaie de comparer les métaphores de Harding et Montrelay. Je me sers encore d'un tableau pour établir cette comparaison.

Esther Harding

Michèle Montrelay

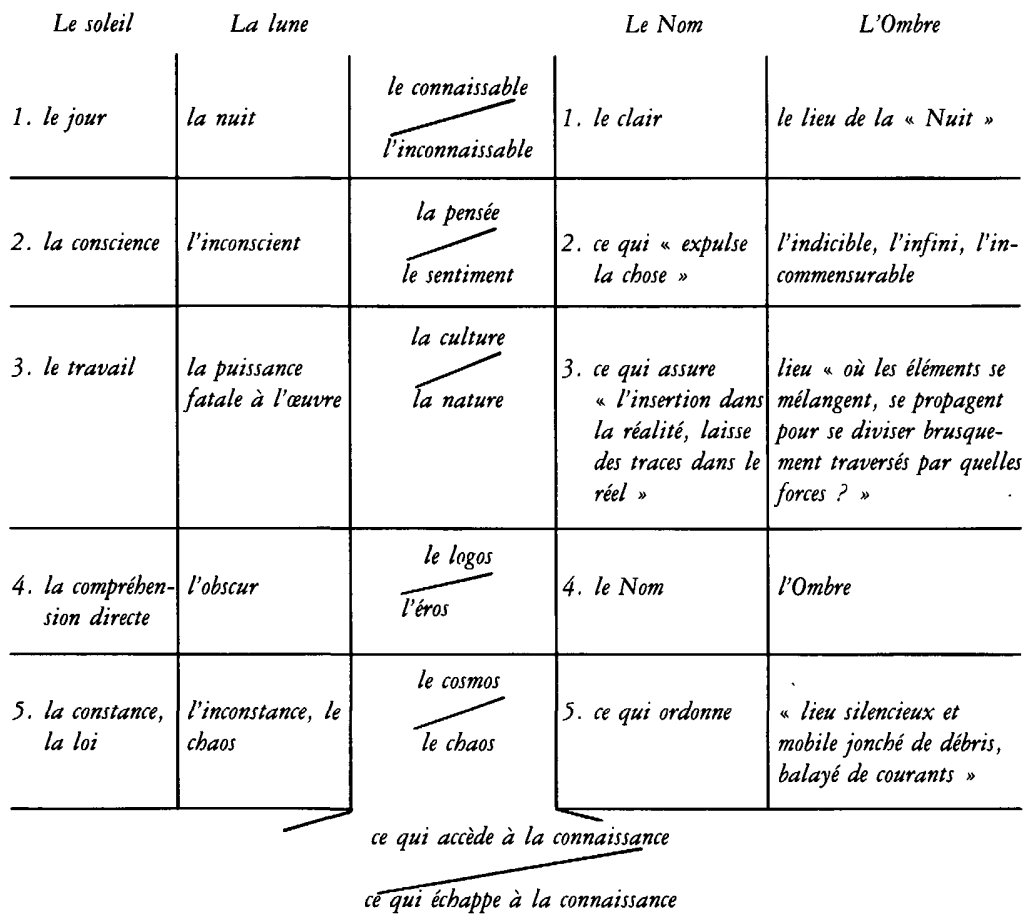

Tout ce jeu d'oppositions utilisé par ces deux psychanalystes aux lieux référentiels si éloignés m'est suspect. Ces différentes désignations peuvent être condensées, à mon avis, dans l'opposition suivante : le masculin est ce qui accède à la connaissance/ le féminin ce qui échappe à la connaissance. 
Comment a-t-on procédé pour parvenir à ce mode de penser, à cette connaissance ? Comment a-t-on réussi à saisir les choses au milieu de leur multiplicité effarante? On a conçu l'Unité, puis plusieurs unités ; et on y a rattaché toutes les multiplicités. Ainsi l'ordre, le cosmos peut être et le multiple désarçonnant est ramené sagement à un Nom-Loi.

Je vois dans ce procédé plusieurs économies intéressantes. La première : l'économie du multiple jugé par l'Un et mis à sa place, ordonné, épuré de tout ce qui fait peur. Le pouvoir et surtout le pouvoir sur le menaçant est instauré, avec sa forme favorite, celle vers laquelle il tend le plus volontiers : une concentration dans peu de mains; ici les mains de l'Un.

Autre économie intéressante : on a pu désigner ce qui peut être connu, c'est-à-dire ce qui peut participer au discours, et ce qui ne le peut pas - mais le discours se permet d'en parler à partir de ses propres références. Cela semble fonctionner à peu près de la sorte : ce qui ne participe pas au discours est contenu dans le discours comme contraire, opposé, en dehors de lui. Donc exclu en quelque sorte d'une exclusion qui est aussi une récupération car il faut veiller à endiguer son action qui risque d'être subreptice.

Essayons de voir comment ce mécanisme peut être appliqué à la question du féminin. Des motifs de peur, d'impérialisme, de goût de pouvoir, des difficultés à saisir la différence, de la facilité à procéder par raisonnement plutôt que par observation et d'autres choses encore ont servi à désigner le féminin comme inaccessible à la connaissance, exclu de celle-ci. Puis a été opérée son inclusion. Le féminin est ce qui s'oppose au masculin, c'està-dire à son discours, à la possibilité d'un discours sur lui autre que poétisant (un discours au statut hors-statut). Et voilà que de rejeté, banni qu'il est, on l'accuse/excuse d'être un éternel échappé annihilant par le fait même sa véritable échappée puisque déjà à l'avance récupérée comme telle.

Est-ce que toutes les différences et surtout les différences qui auraient pu prendre pouvoir, c'est-à-dire recouvrant des personnes ou groupes de personnes ont été ainsi aménagées, dessaisies de leur identité pour n'exister qu'en termes de comparaison, les termes étant toujours déjà, à l'avance hiérarchisés? 
La désignation à partir d'une négation est une habitude que l'on retrouve monotonement : le Noir, celui qui n'est pas blanc, le fou, celui qui n'est pas sain, le pauvre, celui qui n'est pas riche. Et vite, l'on s'aperçoit que l'un des termes possède immédiatement sa référence, son discours, ses lieux de désignation ; l'autre terme ne désignant que son éloignement ou sa proximité au terme référentiel.

Si cette opposition hiérarchisée travaille constamment les concepts peut-on penser les différences de manière égalitaire?

Montrelay nous suggère aussi une lutte entre ces incompatibles. L'Ombre, jouissance saisie par l'Infini de l'Autre s'articule (dans un rapport de lutte ?) au Nom du Père " qui n'est pas donné une fois pour toutes. Qui est à détruire et à expulser, à refaire sans cesse ${ }^{12}$. De cette articulation, de ce Nom qui refoule l'Ombre et permet le signifiant naîtrait une féminité plus complète ? plus intégrée ? Du jeu de cette incompatibilité naîtrait le bénéfice voire l'harmonie de l'affrontement ou de la cohabitation des contraires? Dans quelle mesure peut-il s'effectuer ? Peut-on penser là encore à une certaine androgynie, parler d'une partie masculine de la psyché, d'une partie féminine?

Autre question qui peut être posée à $\mathrm{M}$. Montrelay : pourquoi choisir de refouler la féminité et non pas de la vivre sur " son " mode immédiat, entier, manifeste ; pourquoi demander le verbe à la place du corps ? Parce que l'économie du désir de la femme est instable, obligée à d'incessantes négociations, à des reprises continuelles... Serait-ce le Nom, l'ordre phallogocentrique qui connoterait cette économie de dilapidatrice ou vraiment la féminité gagnerait à être cultivée en ce sens ? En quel nom ? Ce choix de M. Montrelay gagnerait à être expliqué, justifié ou élaboré.

En dernière analyse, on peut se demander s'il est inévitable de parler du féminin et du masculin en ces termes, si l'effet des habitus mentaux sur le corps est tel qu'ils ont ainsi construit cette réalité. Ou encore si l'esprit humain a bien su saisir dès la nuit des temps comment on pouvait parler ce sujet. Ou peut-

12. Montrelay, Michèle, op. cit., p. 145. 
on critiquer cette manière d'ordonner les différences autour d'axes de symétrie, autour de contraires qui se définissent l'un par la négativité de l'autre?

Aussi d'un œil plus infléchi par des visées politiques qu'arrimé à des considérations épistémologiques on peut se demander s'il est judicieux de nous ramener à de telles métaphores ? N'estil pas dangereux de définir un terme par l'infini, l'indicible, l'incommensurable, l'inexplorable ? Cela risque de perpétuer la définition du féminin silencieux, chaotique (etc.) par le masculin maître de la parole et de l'ordre. Ou de nous reporter encore et encore à toutes les mystifications auxquelles oblige ce qui se situe dans l'inconnaissable. Ne se heurtera-t-on pas de nouveau aux images, spectres du féminin : ce qui engage à la fusion profonde et charnelle (où l'on se perd, se détruit) ou ce qui menace de l'horrible castration-mort - ce qui détruit avec duplicité, quoi ! -

Évidemment on peut nous répondre que le savoir doit tenir compte de ce qui lui échappe et ne pas nommer ce qui ne le permet pas. Mais est-ce une position soutenable que de définir comme constituant primordial d'un terme l'indicible et compagnie ? Il me semble que cette position pourrait être exploitée dans tous ses miroitements par des démagogues. Et cela n'a-til pas déjà été fait ?

\section{DE L'ORIGINE DE LA DICHOTOMIE}

La symétrie retourne une chose autour d'un axe, et la présente donc en vis-à-vis, en adversaire, au sens spatial du mot. Elle oppose donc la chose à elle-même, le même au même, et c'est dans cette identité des adversaires qu'il faut trouver l'adversité au second sens, plus seulement spatial, l'bostilité.

Vincent Descombes

On retrouve donc dans les métaphores de Harding et de Montrelay l'opposition d'une première naissance (de l'Obscur, de l'Indéfinissable naît le corps, le désir) provenant du féminin à laquelle succède une seconde naissance (du Nom, de la Lumière surgit ce qui prend place, force dans le réel) attribuée au Père.

Cette opposition possède sa hiérarchie : la première naissance obscure, imprévisible appelle une seconde naissance ras- 
surante, réelle, bien inscrite. Le corps ne prend vie que par l'esprit qu'on lui insuffle. L'on se retrouve devant la vieille idée souvent répétée depuis l'histoire de la création d'Adam, répétée, par exemple dans les termes de la Nature vaste, indomptable qui prend visage, sens quand enfin marquée par la Culture, ou de la Matière qui a besoin d'une Forme.

Quel est le sens de cette hiérarchisation ? On ne naît vraiment que si l'on naît par l'homme et dans son monde ; la première naissance, celle du corps, celle que le féminin, la femme donne est insuffisante. Au fait, y a-t-elle accès à cette seconde naissance, elle qui ne peut pas vraiment atteindre les différents lieux culturels valorisés selon les époques - l'âme, la pensée, la science, le surmoi ? Elle donne naissance à l'homme qui ne se chargera pas de lui donner seconde naissance mais s'ingéniera à lui voler la valeur de la première naissance. L'industrie secondaire a toujours surpassé l'industrie primaire.

Pourquoi ces fantasmes du rapport à l'origine ? Y auraitil un lien entre la connaissance qui s'exerce à travers un jeu d'oppositions et cette origine première qui se presse d'être ennoblie, effacée par une seconde naissance?

Luce Irigaray ${ }^{13}$ pour répondre à cette question, nous propose de relire le mythe de la caverne de Platon comme la méta-

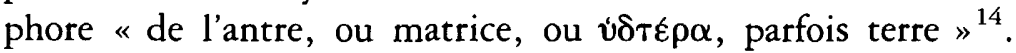
Projet d'interprétation donc reprise de la métaphysique à travers son parachèvement - mais aussi son détournement.

Pourquoi ce désir ? Pour réintroduire dans le " on " asexué du discours philosophique les lieux de la femme et de l'homme, les dire afin de démasquer leur mise sous silence éloquente. Pour dénoncer l'utilisation facile de la symétrie, particulièrement lorsqu'est parlé le féminin comme ce qui n'est pas masculin, son envers négatif, son revers ignoré, oublié, délaissé.

Dans une caverne, des hommes, laissés à l'ignorance. Un homme parfois, un seul à la fois, libéré par quelqu'un d'indéterminé remonte le passage. Sort au soleil qu'il ne peut regarder

13. Irigaray, Luce, Speculum de l'autre femme, Paris, (Minuit), 1974.

14. Irigaray, Luce, op. cit., p. 301. 
en face. Apprend les choses au-delà de leurs apparences. Il ne peut redescendre : il serait tué.

Cela veut dire, nous dit Platon, l'accès à la connaissance. Non, ce n'est pas une progression vers le savoir, nous dit L. Irigaray, c'est le douloureux rapport au séjour intra-utérin duquel on ne peut se détacher mais que l'on veut oublier. Alors on le mime en l'inversant, en le verticalisant, en le faisant pivoter autour d'axes de symétrie. Le centre de cette scène n'est pas le masculin - évidemment encore moins le féminin - mais le masculin tel que projeté à partir de l'idée fixe du maternel, tel que renversé vers une fin puisque ne peut être admise la nécessité de cette origine précise.

De la mère, du corps, de la nature, de l'instinct, les hommes s'en sont coupés par hantise ? par peur ? et dès lors ils se sont enfermés dans la reproduction de cette reproduction qui obsède. Seulement ayant rejeté l'autre, ils se trouvent réduits comme dans la parthénogenèse à reproduire le même. Aussi se retrouvet-on dans l'empire du spéculum, ce moyen favorable à la répétition, à la représentation de la répétition. Mais à cause de cette coupure mal assumée, mal réalisée, de cette rupture qui laisse comme une trace schizoïde s'établit un horizon sur lequel se durcissent oppositions, dichotomies, distinctions claires, catégories ... .

Une seconde naissance a eu lieu; on ne se souviendra que de cette renaissance, de cette origine qui fait taire tout ce qui appartient à l'enfance, aux phantasmes, aux rêves. Désormais le père est reconnu comme engendrant le réel ; à la mère est laissé l'engendrement du fictif.

L'origine, l'origine parlée par le philosophe débute sur «cette rature du commencement " 15 .

Est-ce que L. Irigaray nous raconte à travers ce mythemétaphore-interprétation (?) l'origine de cette irréductible habitude occidentale de penser l'Autre en fonction du Même ? Elle viendrait d'une naissance mal assumée, d'un engendrement maladroitement corrigé. L'imaginaire n'a pas longuement louvoyé.

15. Idem, p. 390 . 
Tout de suite il s'est fixé sur un contraire d'autant plus magnifiquement qu'il devait faire taire, qu'il devait s'empresser de refouler. Somme toute, la fonction de cette lumière est de jeter de l'ombre.

Si l'on tire enseignement de cet . . e écrit, faut-il comprendre que toute pensée procédant par opposition institue une structure hiérarchique? Que dans cette institution tout désir d'égalité s'avère velléitaire ? Que le féminin doit faire méandre parce que peut-être on se baigne toujours dans le même fleuve ; qu'il doit devenir style pour excéder les catégories; qu'il doit changer d'instruments pour se permettre de nouvelles explorations?

De quels styles pourrait-on jouer pour échapper au concept, aux oppositions hiérarchiques ? Y aurait-il moyen d'échapper à notre mode de pensée qui convertit toutes les différences en des oppositions hiérarchisées ? Est-il possible de subvertir ce mode d'appréhension du réel ; ou doit-on conclure que la pensée tyrannisée par elle-même ne peut pas se soustraire à elle et retombe incessamment dans sa reproduction?

POSSIBILITÉS DU PLURIEL

Jacques Derrida ${ }^{16}$ nous propose de passer d'une théorieposition à une théorie-proposition, du dévoilement de la vérité à la kermesse d'interprétations, de la vérité du sens à la notion d'« indécidabilité » qui plane sur chaque affirmation (sur l'affirmation de l'indécidable, bien sûr.).

Il propose de délaisser le concept, de lui juxtaposer la métaphore, le poème, les légendes et mythes, l'éclatement des images (Klossowski) . . . et le reste à découvrir, la restance à assumer.

Faire lâcher la prise de l'herméneutique pour arriver aux différentes propositions de l'interprétation. Chercher un sens oui, car il ne faut rien exclure, mais cette opération herméneutique sur le texte s'ajouterait au même titre à toutes les autres activités transformatrices de l'interprétation.

16. Derrida, Jacques, Éperons, Les styles de Nietzsche, Paris, " Champs ", (Flammarion), 1978. 
$\mathrm{Ne}$ pas se laisser prendre à chercher ce qui est. Rien d'essentialisant. Pas de féminité ou de féminité du féminin ou de sexualité féminine mais l'opération féminine.

$\mathrm{Ne}$ pas oublier dans tout discours le sujet de l'énonciation, son incidence et celles du non-dit, tu ou ignoré.

Ceci amène à valoriser le discours dans ses marges. Le vrai et le faux sont une catégorie insuffisante ; l'indécidable prend la place. Un " savoir » cousu d'indécidables, parcouru de décisions d'auteurs qui ont aussi un rapport inconscient à leur discours.

Non plus l'unité mais la complicité dans les multiples alliances, mouvances, duplicités auxquelles elle peut se rendre.

Non plus la cohérence (comment demander l'absence de contradictions ?) mais la congruence. Ainsi les propositions d'apparence féministe dans l'énorme corpus des propositions antiféministes chez Nietzsche font structure, corps . . .

Derrida nous donne exemple d'une lecture possible de ces mots : «J'ai oublié mon parapluie ».

Que dire de cette phrase entre guillemets écrite par Nietzche puis éditée ? Elle manque de tout contexte signifiant. Et même s'il était retrouvé, quelles certitudes pourrait-on y lire ? L'auteur est toujours adossé à un inconscient qui prend place dans le texte. Et jusqu'à quel point un auteur assume-t-il ce qu'il écrit ?

Ces mots ont une strate de lisibilité : leur sens organisé syntaxiquement. Elle peut donner lieu à des traductions sans perte dans toutes les langues qui disposent d'un certain matériau à des opérations interprétatives plus élaborées (un décryptage psychanalytique, par exemple). Aussi on peut se retrouver devant un jeu parodique du sens, un secret non parce qu'il en détient un mais parce qu'il en manque, un texte codé et encore . . . et encore . . .

Il ne faut pas conclure qu'il faille renoncer au sens. Les limites ne bordent pas le savoir mais le divisent et le traversent.

Cependant, cette proposition laisse subsister une objection. Si l'on pense à travers des métaphores, images, mythes, poèmes, 
et encore . . ., connotés par le jeu des nombreuses hiérarchies, on risque par la multiplicité même de ces discours de masquer, éloigner, nier cette nomination inégalitaire. Peut-être faudraitil opérer, pour l'enrayer ou tout au moins la réduire, une déconstruction systématique du multiple et non pas seulement de l'Un.

\section{**}

Faut-il se résigner à penser que l'égalité est un concept trop nouveau ? Il s'est greffé en nous mais d'une greffe ne réussissant pas encore à créer ce qui lui donnerait statut efficace. S'il est vrai que la fonction crée l'organe comme nous le proposent certaines théories évolutionnistes, on se situe dans un temps historique où la fonction existe, s'impose, perdure (les Grecs ont déjà aménagé un certain espace au concept d'égalité) mais maladroite elle tarde quant à la création de ce qui permettrait son exercice. En quelque sorte, elle serait dans un temps où seul le possible, le dire lui appartiendrait.

La pensée, est-elle vraiment susceptible de nommer avec une " mentalité égalitaire "?

Département de philosophie

Université de Montréal 